\title{
Modified Phoenix Cell for Microstrip Reflectarray Antennas
}

\author{
Sarah A. Ibrahim \\ Callan Institute \\ National University of Ireland Maynooth \\ Co. Kildare, Ireland \\ sibrahim@eeng.nuim.ie
}

\author{
Hany F. Hammad \\ Communications Department \\ German University in Cairo \\ New Cairo, Egypt \\ hany.hammad@guc.edu.eg
}

\begin{abstract}
This paper presents a newly developed modification to the structure of a linearly polarized reflectarray antenna cell, called Phoenix [1]. The idea of Phoenix is based on employing multi-resonators to improve the antenna bandwidth characteristics. Another interesting feature is the phase variation cycle which is characterized by a rebirth capability that was the reason behind the Phoenix name. The new developed modifications resulted in reducing the cell size by $0.16 \lambda_{o}$. In addition to improving the linearity of the phase variation characteristics which in turn reduced the phase errors by $10^{\circ}$ compared to Phoenix. Two reflectarray antennas $\left(200 \times 200 \mathbf{m m}^{2}\right)$ were designed at $9.4 \mathbf{G H z}$ in order to test the new modifications applied. The simulations and measurements indicated an increased antenna directivity for the new modified phoenix reflectarray antenna. Moreover, the measured gain of Phoenix and modified Phoenix at $9.4 \mathbf{G H z}$ is $17.8 \mathrm{~dB}$ and $20.9 \mathrm{~dB}$, respectively.
\end{abstract}

\section{INTRODUCTION}

Microstrip reflectarray antenna is a recent technology which was developed as an alternative for parabolic reflectors, since it combined the best features of the conventional parabolic reflectors together with the low-profile printed microstrip antenna technology. It consists of a two dimensional array of microstrip elements which is directly fed by a horn antenna [2]. The idea of reflectarray antennas is based on applying phase compensation techniques to the design of each cell inside the array to make for the spatial phase delay of the incident electric field encountered by each cell so as to achieve an in-phase re-radiated far-field beam from the reflecting surface. There are several approaches which have been recently developed to achieve a planar phase front for different polarization. The most common approach was developed using different size of radiating patches, dipoles or rings to produce a cophasal far field beam. In 1998, Huang and Pogorzelski reported an efficient way of producing phase variations using identical patches with variable angular rotations [3].

\section{Modified Phoenix Cell Design}

Phoenix cell design is based on employing multi-resonators on the same layer of the substrate, thus it improves the bandwidth characteristics compared to most of the reflectarray elements while maintaining a low-profile structure. Phoenix cell was designed to operate at a center frequency of 16.5 $\mathrm{GHz}$ [2]. The structure of the phoenix cell consists of a square resonating inner patch whose dimension (Lp) is equal to $\lambda_{o} / 8$,

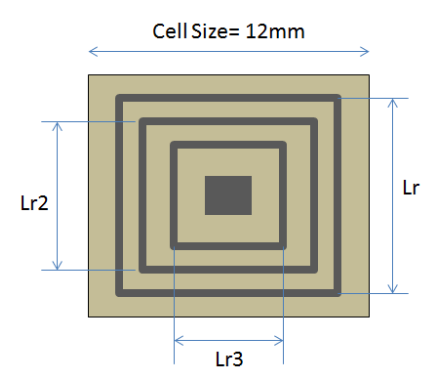

(a)

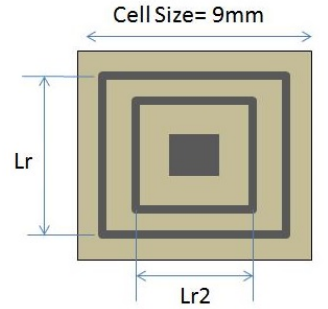

(b)
Fig. 1. a- Phoenix cell structure after the $1^{\text {st }}$ modification, b- Phoenix cell structure after the $2^{\text {nd }}$ modification

surrounded by a microstrip resonating ring as seen in Fig.1-a. The substrate used is Rogers Duroid whose $\varepsilon_{r}=2.2$ and the substrate thickness is $0.254 \mathrm{~mm}$. The ground layer is hanged above the substrate at a height of $6 \mathrm{~mm}\left(0.33 \lambda_{o}\right)$. The interelement spacing for the array is $12 \mathrm{~mm}\left(0.66 \lambda_{o}\right)$. The phase variation cycle is based on varying the length of the outer resonating ring (Lr), as it starts with $\mathrm{Lr}=\mathrm{Lp}$ where $\mathrm{Lr}$ is increased till it exceeds the cell size driving the cell structure to the initial point of the cycle. A modification was applied on the phoenix cell in a way to extend the phase range covered by the element, where an extra resonating ring was added to the structure of the cell as shown in Fig.1-b.

\section{Phoenix Simulation}

Simulations were carried out for both Phoenix and the modified Phoenix using the TEM waveguide setup [4]. The phase variation of the reflection coefficients from the surface of both cells at each different $\mathrm{Lr}$ dimension were monitored, and the modified phoenix has found to enlarge the range of the phase shift achieved up to $540^{\circ}$ instead of $300^{\circ}$ as seen in Fig.2. Since the full phase range is defined by only $360^{\circ}$, therefore the size of the modified phoenix can be reduced, and a further modification was applied having the cell size reduced by $0.16 \lambda_{o}$. Fig. 3 shows the simulated phase variation of the new Phoenix after the reducing the cell size.

\section{EXPERIMENTAL VAlidation}

In order to experimentally evaluate the performance of the new Phoenix cell, two square reflectarrays were designed 


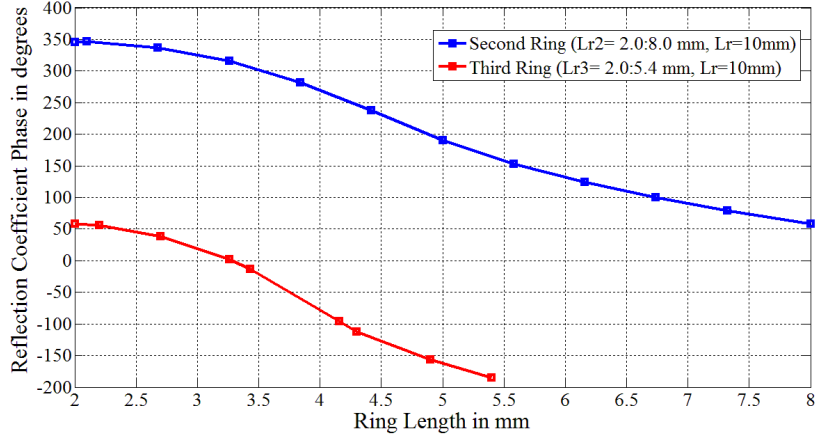

Fig. 2. The simulated phase variation of Phoenix cell after the $1^{\text {st }}$ modification.

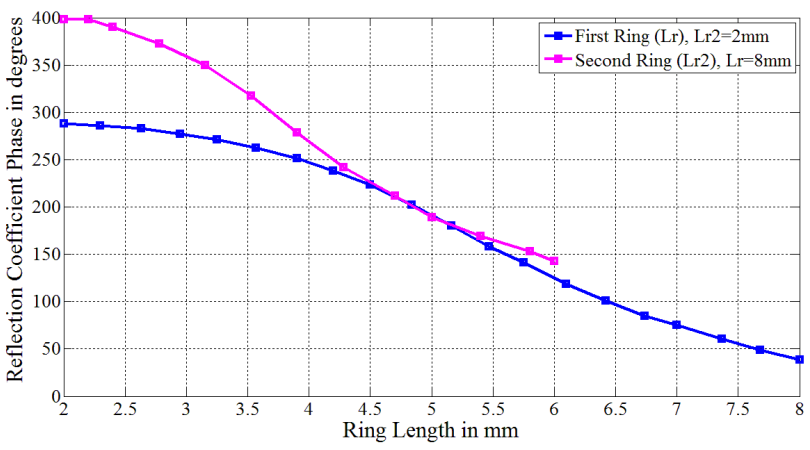

Fig. 3. The simulated phase variation of Phoenix cell after the $2^{\text {nd }}$ modification.

using Phoenix and the newly modified Phoenix. The size of each array is $200 \times 200 \mathrm{~mm}^{2}$. A rectangular horn antenna was employed as a feeding antenna for both reflectarrays which were designed to operate at $9.4 \mathrm{GHz}$ in order to fit the available lab equipments. The horn was hanged above the array at a distance equal to the focal point of the array aperture $(0.6 \mathrm{D}$, where $\mathrm{D}$ is the diameter of the array).

Fig.4 shows the fabricated reflectarrays together with the feeding horn attached. Fig.5 shows the measured pattern of both antennas which indicates having a lower sidelobe level for the new Phoenix and an increased directivity by $5 \mathrm{dBi}$. Fig. 6 shows the measured gain of both antennas over around 10 bandwidth.

\section{CONCLUSion}

A new modified Phoenix reflectarray cell was developed, simulated and compared with the previously designed Phoenix. The new modifications reduced the cell size by $0.16 \lambda_{o}$ and enlarged the delivered phase range by $60^{\circ}$. Moreover it improved the linearity of the phase variation cycle. The measured new Phoenix antenna proved to increase the antenna gain by more than $2.5 \mathrm{~dB}$ over a 10 bandwidth.

\section{ACKNOWLEDGMENT}

The authors wish to acknowledge the assistance and support of CTVR- The Telecommunications Research Center in cooperation with the German University in Cairo.

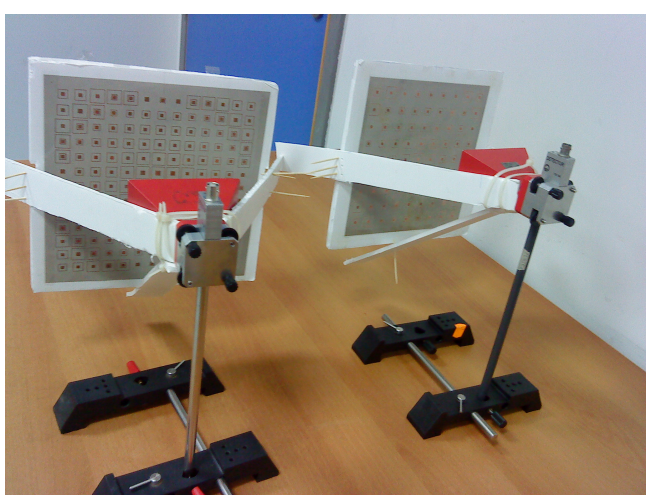

Fig. 4. The fabricated Phoenix reflectarray antenna.

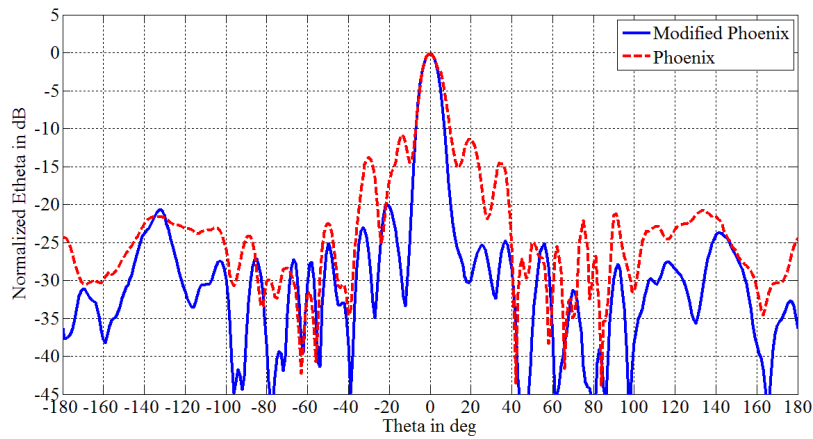

Fig. 5. The measured patterns of both Phoenix antennas.

\section{REFERENCES}

[1] L. Moustafa, R. Gillard, F. Peris, R. Loison, H. Legay, and E. Girard, "The Phoenix Cell: A New Reflectarray Cell With Large Bandwidth and Rebirth Capabilities," IEEE Trans. Antennas And Wireless Propagation Letters, vol. 10, 2011.

[2] D. M. Pozar, S. D. Targonski, and H. D. Syrigos, "Design of Millimeter Wave Microstrip Reflectarrays," IEEE Trans. Antennas Propag., vol. 45, no. 2, February 1997.

[3] J. Hunag and R. J. Pogorzelski, "A Ka-band microstrip reflectarray with variable rotation angles," IEEE Trans. Antennas Propag., vol. 46, no.5, pp.650-657, May 1998.

[4] A. Wahid, M. K. A. Rahim and F. Zubir, "Analysis of Dual Layer Unit Cell with Minkowski Radiating Shape for Reflectarray Antenna on Different Substrate Properties," IEEE AsiaPacific Conference on Applied Electromagnetics, 2010.

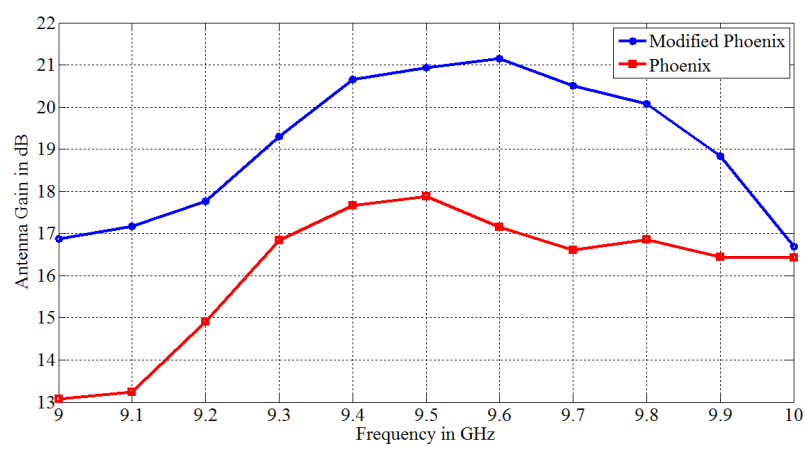

Fig. 6. The measured gain of over $10 \%$ bandwidth. 